\title{
Involving service users in the recruitment of mental health staff: the Service User Informed Tool for Staff Selection (SUITSS)
}

Ángel Sánchez-Bahíllo, ${ }^{1}$ Claire Davis, ${ }^{2}$ Richard Onyon, ${ }^{3}$ Jan Birtle ${ }^{4}$

The Psychiatrist (2012), 36, 133-136, doi: 10.1192/pb.bp.111.035030

${ }^{1}$ Neurociencia Clínica y Experimental (NICE-CIBERNED), Universidad de Murcia, Spain; ${ }^{2}$ Avon and Wiltshire Mental Health Partnership NHS Trust, Bath, UK; ${ }^{3}$ Coventry and Warwickshire Partnership Trust, Coventry, UK; ${ }^{4}$ Clinical Director Pathways for Personality Disorder, Birmingham and Solihull Mental Health NHS Foundation Trust, Birmingham, UK

Correspondence to Ángel SánchezBahíllo

(angelsanchezbahillo@hotmail.com) First received 21 Apr 2011, final revision 10 Oct 2011, accepted 17 Oct 2011

\begin{abstract}
Aims and method Service user involvement in the development of services is a fundamental aim of the National Health Service (NHS). However, a structured and quantifiable approach to their involvement in the recruitment of NHS staff is still lacking. In this study, we used service-user focus groups within a therapeutic community for people with personality disorder in order to develop the Service User Informed Tool for Staff Selection (SUITSS).
\end{abstract}

Results We enabled service users to develop SUITSS as a Likert scale with which to define relevant staff characteristics and rate applicants according to them, informing the staff-selection process. The tool has a semi-quantitative format that allows to test whether applicants with higher ratings are actually appointed by selection panels or not.

Clinical implications This new tool provides an approach to enhancing service user contributions to staff recruitment. It may be adapted and refined for use in a range of services, with local input from service users, following the approach described here.

Declaration of interest None.
The involvement and empowerment of service users is a fundamental aim of the National Health Service (NHS), ${ }^{1}$ and their participation in the recruitment of staff is an essential component of this. However, a structured and quantifiable approach to the contribution of service users to staff selection is still lacking. As highlighted by Bhui et $a l^{2}$ service user involvement can include user representation on planning groups, involvement in designing new services, participation in staff training and involvement in staff selection. Nonetheless, attempts to incorporate service users' views in a consistent and inclusive manner can be subject to distortion, as demonstrated by Crawford et $a l^{3}$ who found that service users who take part in surveys do not adequately represent their community.

Within mental health services the importance of service user involvement in service development and design is particularly important, as ensuring they have a voice provides a therapeutic opportunity to counter the stigma, disempowerment and marginalisation experienced by many people with mental health problems. This has potential benefits not only for service quality improvement but also for individuals as part of their recovery journey. Failure to incorporate service users' perspectives in the recruitment of the staff who are going to work with them deprives the NHS of the unique voice of those on the receiving end of the care it provides and opens it to the risk of becoming a technocracy, disempowering and alienating those it aims to help. This would reinforce the perceived traditional paternalistic approach of mental health systems towards service users, undermining their autonomy. ${ }^{4}$ However, if the contribution and expectations of service users in recruitment are not clearly defined, there are risks that this may become just a tokenistic well-meaning exercise.

The lack of a system to involve service users in staff recruitment is especially important for people diagnosed with personality disorder ${ }^{5}$ and the structure of a therapeutic community in which these service users are sometimes treated is ideal for the development of a process that could then be used widely across health services. Service users already participate in the recruitment of mental health staff and on occasion individual service users take part in selection panels. ${ }^{6}$ However, this approach is not structured, and asking service users to be part of a selection panel without being clear about the role and contribution they provide can be problematic. In an example witnessed by one of the authors, a service user who had been subjected to sexual abuse as a child stated 'we can't have him, I don't trust him, he's an abuser', leaving the interview panel very split about how to respond and to what extent recruitment should support this view, instinctive as it was but also reflecting high sensitivity to previous trauma. There was a disconnect between the rigorous selection process led by 
staff and the risk that service user contributions would either be overemphasised or minimised.

A PubMed search on the 9 February 2011 did not find any articles reflecting initiatives to involve service users in the recruitment of mental health staff beyond the participation of an individual in a selection panel. The need to empower NHS service users is more acute in personality disorder services as the Department of Health acknowledges that 'people with a primary diagnosis of personality disorder are frequently unable to access the care they need from secondary mental health services'. ${ }^{7}$ Thus, the involvement of this particular group of service users in the provision of their care is especially important. A therapeutic community provides an ideal environment in which to gather service user views. Each therapeutic community, although formed by disparate individuals, develops a common 'matrix' of relationships and communications as a result of its history and circumstances, and a specific 'culture, ${ }^{8}$ that supersedes the individuals and expresses a group view over different issues. This consensus, along with the essential negotiation to reach it, goes beyond aggregates or 'averages' obtained from approaching individual service users who may not adequately represent their community $^{9}$ and ensures the representativeness of its findings.

This study describes a methodological approach to purposefully processing service users' views in order to develop and pilot a structured tool (Service User Informed Tool for Staff Selection, SUITSS) for gathering the views of the community of service users on NHS staff applicants during the process of recruitment. Given the paucity of studies investigating the effect of service users' involvement in practice, ${ }^{10}$ we intended to design SUITSS as a tool that enabled service users to (a) define parameters that they considered important and (b) to quantify these parameters by giving broad ratings for applicants that were measurable.

\section{Method}

\section{Development of the tool}

In an NHS personality disorder service composed of two therapeutic communities (a residential therapeutic community and a day therapeutic community) we formed two focus groups, each comprising members of one of the therapeutic communities and led by the investigators. These focus groups worked sequentially on the development of the tool as follows.

a The residential therapeutic community focus group discussed the characteristics they would like to see in a staff member and identified which of those characteristics should be rated in the process of selection.

b The investigators enabled the focus groups to organise these characteristics in a Likert scale that could be used to rate staff applicants.

c This draft tool was discussed within the day therapeutic community focus group and refined to constitute the working pilot version of SUITSS.

\section{Use of the tool}

Although the development of SUITSS took place across the service in order to identify staff characteristics that were relevant to work with this service user population, its application was specific to the residential therapeutic community to which staff were being recruited.

It was the standard practice of the residential therapeutic community to invite all short-listed staff for a day visit to the community. During this visit, staff applicants would meet service users and professionals and take part in the therapeutic programme. Service users had the opportunity to discuss their views on staff applicants in a group setting. Following the implementation of SUITSS, on the day after staff applicants' visit, service users were asked to complete the SUITSS. Then, an average of the ratings, along with a summary of comments, was forwarded to the staff selection panel to inform the recruitment process. The investigators observed this process and also collected feedback from staff applicants who participated. Due to organisational difficulties, it was not possible to gather formal feedback from the service users who utilised the tool during the development stages, although it was recognised that this would have been preferable.

\section{Evaluation of its impact}

The SUITSS was designed in such a way as to allow comparisons between the ratings of the different items of the SUITSS for those staff applicants deemed appointable by the selection panel and those who were not. This comparison would help to evaluate the impact of the service user's voice as expressed through the SUITSS on the final selection and to better understand the correlation, or lack of it, between the views of service users and those of the selection panels. At this pilot stage of the project, our main focus was the process of the development of the tool and any evaluation of this correlation was not made, primarily due to the large numbers of recruitment rounds that would be required to ensure statistical significance.

\section{Results}

\section{Development of the tool}

The outcome of the project has been to develop a process that enables service users to build a tool through which they are able to provide a formal input to the staff selection process. Figure 1 shows the tool as it was designed by service users and piloted with staff applicants. The role of the investigators was to support and empower the community in finding their voice and thus their choice of words to express their views. The investigators described having to hold back from making suggestions to ensure that the tool that was developed authentically reflected this emphasis.

\section{Use of the tool and evaluation of its impact}

The tool was used by members of the residential therapeutic community to evaluate three applicants to a staff post within that community. Service users participated in this process and engaged well in all stages of the development of 
Service User Informed Tool for Staff Selection

Name of staff applicant:

The therapeutic community should rate the staff applicants according to the following parameters.

\begin{tabular}{|c|c|c|c|c|c|c|}
\hline & $\begin{array}{c}\text { Very little } \\
1\end{array}$ & $\begin{array}{c}\text { Little } \\
2\end{array}$ & $\begin{array}{c}\text { Medium } \\
3 \\
\end{array}$ & $\begin{array}{c}\text { Some } \\
4\end{array}$ & $\begin{array}{c}\text { A lot } \\
5\end{array}$ & Comments \\
\hline \multicolumn{7}{|l|}{ Genuineness } \\
\hline \multicolumn{7}{|l|}{ Respect for boundaries } \\
\hline \multicolumn{7}{|l|}{ Humility } \\
\hline \multicolumn{7}{|l|}{ Listening skills } \\
\hline \multicolumn{7}{|l|}{ Empathy } \\
\hline \multicolumn{7}{|l|}{ Capacity to engage } \\
\hline \multicolumn{7}{|l|}{ Sense of humour } \\
\hline \multicolumn{7}{|l|}{ Capacity to be active } \\
\hline \multicolumn{7}{|l|}{ Capacity to keep calm in a crisis } \\
\hline \multicolumn{7}{|l|}{$\begin{array}{l}\text { Capacity to challenge and accept } \\
\text { challenging }\end{array}$} \\
\hline \multicolumn{7}{|l|}{$\begin{array}{l}\text { Appropriateness to complement } \\
\text { current staff team }\end{array}$} \\
\hline Global appointability for the post & & & & & & \\
\hline
\end{tabular}

Post applied to:

Date of evaluation:

Place of evaluation: .

Number of people in the evaluation group:.

Comments:

Fig 1 Service User Informed Tool for Staff Selection (SUITSS) as prepared by service users in the study.

the tool. They were interested and involved in discussions about the selection of parameters and in the use of ratings. In addition, this provided a useful structure for discussion about the candidates, which assisted in decisions about appointment. Staff reported improvements in the process of engagement with service users in that they were able to have more focused discussion. Overall, there was widespread agreement that this provided a more transparent and robust selection process. There were limitations in the formal feedback from service users (see Discussion).

\section{Discussion}

We successfully developed a structure that enables service users to build a tool through which to express their views on staff recruitment, thus informing and improving the quality of the recruitment process. Such involvement empowers those who are on the receiving end of services and engages the community in participation in the development of services as recommended by Elstad \& Eide. ${ }^{11}$ The implementation of this tool was conducive to developing the service as a flexible, accessible and dynamic organisation and enriched its capacity to reflect and to employ the most suitable staff taking into account the view of service users. Staff members, having had their future clients involved in their selection process can also feel empowered and confident regarding their suitability for their post and the interaction with service users it requires. Unfortunately, as the research aimed to provide minimal disruption to therapeutic engagement, we could not gather service users' views on their experience of using the tool through additional focus groups. This is an aspect requiring further exploration.

\section{Applicability of the tool}

Although the tool shown in Fig. 1 was tailored to meet the particular needs of a residential therapeutic community for people with personality disorder, SUITSS could be utilised as a basis from which local variations, informed by service user consultation, could be tailored to a range of different services. This includes mental health, non-mental health services, the private sector and its use overseas in languages other than English. Furthermore, as services undergo redesign, SUITSS could be reviewed and modified as required. It would also be informative to test to what extent the tool is relevant to a range of cultures, whether it 
is describing generalised important factors in staff selection and to what extent these may be culturally and linguistically bound. A proposed pilot of a version of SUITSS in Spain will begin to address these issues.

Clearly, it is important to be mindful that service users' views should not override all other considerations in the staff-selection process. An adequately balanced combination of the views of service users, the therapeutic community, staff, local and national policy and a realistic consideration of resources should guide staff selection in particular and service development in general.

Although individual service users might have very disparate views about the characteristics of the health professional they would like to work with, the development of a group perspective where these views are balanced and cross-fertilised is essential to deliver a coherent perspective that represents the view of the target population. This is relatively accessible in a therapeutic community, where group exploration is an important therapeutic component and where SUITSS was developed and piloted, but could be more challenging in settings where service users have limited contact with each other, namely in out-patient settings. In these cases, the engagement of service users to form a cohesive 'work group'12 able to muster group thinking, would require interventions prior to the application of SUITSS, for example through the implementation of focus groups.

The group process is susceptible to deviation from specific goals and can move into unhelpful or destructive modes, ${ }^{13}$ and the development of SUITSS in a group setting has to consider the potential for negative influences to be expressed. These could take the form of unfair criticism of applicants, deliberate recommendations to recruit unsuitable applicants as a sabotaging manoeuvre, etc. During our project, we could observe individual members completing the SUITSS in isolation or in ways suggesting they were expressing their personal view rather than trying to interpret that of the community. Also, it appeared that some service users' ratings could be somewhat speculative, such as rating an applicant's capacity to keep calm in a crisis without having seen the applicant in a crisis situation. We would recommend that this process be supervised by professionals who are familiar with group dynamics and who can both identify and challenge these processes.

\section{Evaluation of its impact}

One of the virtues of SUITSS is that, in providing a semiquantitative measure of the service users' views on staff applicants, it permits the auditing of its impact and the correlation between service user recommendations and the outcome of recruitment. The large number of staff applicants that need to be rated to determine whether selection panels' decisions correlate in any way with service users' perspectives mean that this will need to be addressed in multicentre studies.

In conclusion, this study has demonstrated that the engagement of service users in the recruitment of staff in the setting of a therapeutic community was considered to be significantly enhanced by the development of a systematised tool, SUITSS. The development of the tool actively involved service users in defining parameters that they considered important in staff that were to work with them, delivering services. The study then progressed to utilising the tool in staff selection, with a positive impact reported in the quality of discussion about candidates. In addition, the candidates felt positive that their selection had involved service users and this assisted them in feeling confident about taking up their roles. As summoning service users to new focus groups would interfere with the tight therapeutic programme in a residential therapeutic community, a fuller evaluation of service user views following recruitment using SUITSS was not incorporated into this initial study. Neither was the tool redeveloped or tested out in other mental health or cultural settings. Although this pilot study was limited, we intend to further test and refine these initial promising findings in the field.

\section{About the authors}

Ángel Sánchez-Bahillo is a researcher at the Neurociencia Clínica y Experimental (NICE-CIBERNED), Facultad de Medicina, Universidad de Murcia, Spain. Claire Davis is a specialty doctor in psychiatry at the Avon and Wiltshire Mental Health Partnership NHS Trust, Bath, UK. Richard Onyon is a consultant psychiatrist at the Coventry and Warwickshire Partnership Trust, Coventry, UK. Jan Birtle is a consultant medical psychotherapist, Clinical Director Pathways for Personality Disorder, Birmingham and Solihull Mental Health NHS Foundation Trust, Birmingham, UK

\section{References}

1 Department of Health. The NHS Constitution for England. Department of Health, 2009.

2 Bhui K, Aubin A, Strathdee G. Making a reality of user involvement in community mental health services. Psychiatr Bull 1998; 22: 8-11.

3 Crawford MJ, Aldridge T, Bhui K, Rutter D, Manley C, Weaver T, et al. User involvement in the planning and delivery of mental health services: a cross-sectional survey of service users and providers. Acta Psychiatr Scand 2003; 107: 410-4

4 Hansen T, Hatling T, Lidal E, Ruud T. The user perspective: respected or rejected in mental health care? J Psychiatr Ment Health Nurs 2004; 11: 292-7.

5 World Health Organization. The ICD-10 Classification of Mental and Behavioural Disorders: Clinical Descriptions and Diagnostic Guidelines. WHO, 1992

6 Bhardwaj A, Kathane R. Involvement of a young service user in the appointment of a doctor. Psychiatrist 2009; 33: 439-40

7 National Institute for Mental Health in England. Personality Disorder: No Longer a Diagnosis of Exlusion. Policy Implementation Guidance for the Development of Services for People with Personality Disorder: 5. NIMHE, 2003.

8 Foulkes SH. The Group as a Matrix of the Individual's Mental Life. Selected Papers. Karnac, 1990

9 Gerkensmeyer JE, Austin JK. Development and testing of a scale measuring parent satisfaction with staff interactions. J Behav Health Serv Res 2005; 32: 61-73.

10 Repper J, Breeze J. User and carer involvement in the training and education of health professionals: a review of the literature. Int J Nurs Stud 2007; 44: 511-9.

11 Elstad TA, Eide AH. User participation in community mental health services: exploring the experiences of users and professionals. Scand $J$ Caring Sci 2009; 23: 674-81.

12 Bion WR. Experiences in Groups. Tavistock, 1961.

13 Nitsun M. The Anti-group: Destructive Forces in the Group and their Creative Potential. Routledge, 1996. 